Evaluar.

2003, n 3 (julio)
Laboratorio de Evaluación Psicológica y Educativa. Facultad de Psicología Universidad Nacional de Córdoba (Argentina). ISSN N¹667-4545

\title{
La Evaluación Del Trastorno Por Estrés Postraumático: Aproximación A Las Propiedades Psicométricas De La Escala De Trauma De Davidson
}

\author{
Villafañe, A. *,. Milanesio, M. S. *, Marcellino, C. M., \\ Amodei, C. ** \\ * Facultad de Psicología. Universidad Nacional de Córdoba \\ ** Laboratorio B/ Psicobiología Experimental. Facultad de Psicología. Universidad Nacional de Córdoba
}

Resumen: Debido a la alta comorbilidad que presenta el Trastorno por Estrés Postraumático (TEPT), se considera relevante hacer una aproximación a las propiedades psicométricas de la Escala de Trauma de Davidson-DTS, que indaga los síntomas del trastorno, para determinar si la misma es un instrumento válido de diagnóstico. Para ello se tomaron dos muestras de sujetos entre 18 y 20 años, una conformada por estudiantes de Psicología de la UNC y otra por estudiantes de diferentes instituciones educativas de Río Tercero. Se administraron cinco instrumentos de autoinforme, dos evalúan síntomas de TEPT (DTS e IES-R), uno de depresión (Beck), un cuestionario de acontecimientos traumáticos y los STAIs, para síntomas de ansiedad. Como conclusión final la Escala de Trauma de Davidson cuenta con propiedades psicométricas aceptables para evaluar el Trastorno por Estrés Postraumático.

Palabras Clave: Trastorno por Estrés Postraumático o TEPT - Propiedades Psicométricas-

Escala de Trauma de Davidson (DTS)

\section{Introducción}

Se consideró relevante llevar a cabo un estudio sobre las propiedades psicométricas de la escala de trauma de Davidson (DTS) debido a que los instrumentos psicométricos pueden constituir, para el clínico, una herramienta útil en la evaluación diagnóstica, sin descartar el uso de entrevistas clínicas.

Cabe mencionar también que el trastorno por estrés postraumático (TEPT) presenta una alta tasa de comorbilidad con otros trastornos por ansiedad (fobia simple, fobia social, trastorno de ansiedad generalizada y trastorno de pánico), trastornos afectivos (depresión mayor, distimia y manía) y con el abuso de sustancias (alcohol y drogas), según los datos reportados por los estudios epidemiológicos del National Comorbidity Survey, el 
Epidemiological Catchment Area Survey y el estudio de Breslau (Helzer y cols., 1987; Kessler y cols., 1995; Breslau y cols., 1991). Por tal razón, realizar un correcto diagnóstico, implica brindar un tratamiento adecuado.

La DTS (Escala de Trauma de Davidson) es un instrumento psicométrico que está constituido por 17 ítems que corresponden y evalúan cada uno de los 17 síntomas recogidos en el DSM IV. Esta escala evalúa la gravedad y frecuencia de los síntomas del trastorno en individuos que han experimentado un trauma.

La DTS posee propiedades psicométricas aceptables. A continuación se exponen los resultados obtenidos por los autores (Davidson, Book, Colket y cols.; 1997) con distintas muestras de pacientes. En total son 353 pacientes que han sido víctimas de distintos traumas: violación ( 78 mujeres), veteranos de guerra (110 varones), víctimas del huracán Andrew (53 individuos) y 102 sujetos supervivientes de diversos acontecimientos traumáticos que participaban en un ensayo clínico multicéntrico. Los resultados obtenidos fueron los siguientes:

Consistencia interna: el coeficiente $\alpha$ de Cronbach para el total del instrumento fue de 0.99; para la subescala de frecuencia, 0.97; para la subescala de gravedad, 0.98.

Fiabilidad test - retest (intervalo 2 semanas). El coeficiente de correlación para el total del instrumento fue de 0,87. Por factores (correspondientes a cada criterio diagnóstico para el trastorno, según el DSM IV) los coeficientes obtenidos fueron: criterio $\mathrm{B}, 0,83$; criterio $\mathrm{C}, 0,78$, y criterio $\mathrm{D}, 0,83$.

Estructura factorial: Se determinó la existencia de dos factores; uno de gravedad que explicaba el $24.79 \%$ de la varianza y otro formado por ítems de intrusión, evitación y embotamiento, que tan sólo explicaba el 1.34\% de ésta. El mismo análisis sólo con los datos de los pacientes que presentaban un trastorno por estrés postraumático, reveló la existencia de seis factores que explicaban el $26.91 \%$ de la varianza y que se asemejaban al grupo de síntomas del trastorno.

Validez convergente: El coeficiente de correlación de la puntuación total de la DTS con las puntuaciones totales en la CAPS (Clinician Administered PTSD Scale) fue de $\mathrm{r}=.78$; en la IES (Impact of Event Scale) fue de $\mathrm{r}=64$, y en el SCL-90R (Symptom Check List) fue de $r=.66$.

Validez divergente: Se calculó mediante la correlación con la escala de neuroticismo de Eysenck obteniendo un coeficiente de correlación de $\mathrm{r}=.04$. 
Capacidad para discriminar: Utilizando como patrón estándar los diagnósticos del SCID -Structured Clinical Interview for DSM IV- (de un total de 129 pacientes entrevistados, 67 presentaban el trastorno y 62 no) se encontraron diferencias significativas entre las puntuaciones obtenidas en la DTS por los pacientes diagnosticados con el trastorno de estrés postraumatico y los que no cumplían con los criterios para el diagnóstico del mismo [62 (38) frente a 15.5 (13.8), p<0.0001]. La DTS también fue capaz de discriminar entre los cinco grupos de gravedad establecidos mediante la PhysicianRated Global Assessment of Severity (GASP); se encontraron diferencias estadísticamente significativas entre las puntuaciones en la DTS de los cinco grupos de gravedad [mínima = 14 (13.8); subclínica, 41.7 (28.1); clínica, 78.5 (27.1); grave, 108.5 (15.4), y muy grave, $114(8.4)]$.

La misma escala fue validada por Bobes García y Calcedo-Barba (2000) en un proyecto multicéntrico español, demostrando poseer unas propiedades psicométricas muy aceptables. Los resultados obtenidos fueron los siguientes:

Consistencia interna: El coeficiente $\alpha$ de Cronbach para el total del instrumento fue de 0.89 , y para los tres factores, correspondientes a cada uno de los criterios diagnósticos para el trastrono, según el DSM IV; fueron: criterio B, 0.83; criterio C, 0.74 , y criterio $\mathrm{D}, 0.76$.

Fiabilidad test-retest (intervalo 2 semanas). El coeficiente de correlación para el total del instrumento fue de 0.87 . Por factores, los coeficientes obtenidos fueron: criterio $\mathrm{B}, 0.83$; criterio $\mathrm{C}, 0.78$, y criterio $\mathrm{D}, 0.83$.

Validez convergente: El coeficiente de correlación entre las puntuaciones de la DTS total y las puntuaciones de la CGI (Clinical Global Impression) fue de 0.62. Por factores los coeficientes fueron: criterio $\mathrm{B}, 0.47$; criterio $\mathrm{C}, 0.64$, y criterio $\mathrm{D}, 0.54$

Validez discriminante: Los pacientes con TEPT mostraron puntuaciones significativamente más elevadas que los controles. Los pacientes considerados graves en la CGI obtuvieron puntuaciones significativamente superiores a los considerados leves por la DTS.

Trastorno por Estrés Postraumático: Descripción

Se ha denominado trastorno por estrés postraumático, al repertorio de reacciones que manifiesta un individuo luego de haber estado expuesto (se incluye aquí el haber 
experimentado, que le hayan contado o que haya visto) a un evento altamente estresante ante el cual ha respondido con temor u horror intensos. El elemento crítico que torna traumático a un suceso es la evaluación subjetiva de la víctima acerca de cuán desamparada o amenazada se ha sentido, es decir, las interpretaciones referidas al significado que se le atribuyen al mismo. La repercusión del suceso traumático se plasma en el interior del organismo, cronificándose y modificando su neurobiopsicología (Van der Kolk \& McFarlane, 1996).

Para una mayor comprensión del trastorno por estrés postraumático, se presentan a continuación los criterios diagnósticos.

Criterios Diagnósticos para el TEPT, según el DSM-IV (APA, 1994).

A. La persona ha estado expuesta a un acontecimiento traumático en el que han existido (1) y (2):

(1) la persona ha experimentado, presenciado o le han explicado uno (o más) acontecimientos caracterizados por muertes o amenazas para su integridad física o de los demás

(2) la persona ha respondido con temor, una desesperanza o un horror intensos. Nota: En los niños estas respuestas pueden expresarse en comportamientos desestructurados o agitados.

B. El acontecimiento traumático es reexperimentado persistentemente a través de una (o más) de las siguientes formas:

(1) recuerdos del acontecimiento recurrentes e intrusos que provocan malestar y en los que se incluyen imágenes, pensamientos o percepciones. Nota: En los niños esto puede expresarse en juegos repetitivos donde aparecen temas o aspectos característicos del trauma.

(2) sueños de carácter recurrente sobre el acontecimiento, que producen malestar. Nota: En los niños puede haber sueños terroríficos de contenido irreconocible.

(3) el individuo actúa o tiene la sensación de que el acontecimiento traumático está ocurriendo (se incluye la sensación de estar reviviendo la experiencia, ilusiones, alucinaciones y episodios disociativos de "flashbacks", incluso los que aparecen al despertarse o al intoxicarse). Nota: Los niños pequeños pueden reescenificar el acontecimiento traumático específico.

(4) malestar psicológico intenso al exponerse a estímulos internos o externos que simbolizan o recuerdan un aspecto del acontecimiento traumático.

(5) respuestas fisiológicas al exponerse a estímulos internos o externos que simbolizan o recuerdan un aspecto del acontecimiento traumático.

C. Evitación persistente de estímulos asociados al trauma y embotamiento de la reactividad general del individuo (ausente antes del trauma) tal y como indican tres (o más) de los siguientes síntomas:

(1) esfuerzos para evitar pensamientos, sentimientos o conversaciones sobre el suceso traumático 
(2) esfuerzos para evitar actividades, lugares o personas que motivan recuerdos del trauma

(3) incapacidad para recordar un aspecto importante del trauma

(4) reducción acusada del interés o la participación en actividades significativas

(5) sensación de desapego o enajenación frente a los demás

(6) restricción de la vida afectiva (p. Ej., incapacidad para tener sentimientos de amor)

(7) sensación de un futuro desolador (p. Ej., no espera obtener un empleo, casarse, formar una familia o, en definitiva, llevar una vida normal).

D. Síntomas persistentes de aumento de la activación (arousal) (ausentes antes del trauma), tal y como indican dos (o más) de los siguientes síntomas:

(1) dificultades para conciliar o mantener el sueño

(2) irritabilidad o ataques de ira

(3) dificultades para concentrarse

(4) hipervigilancia

(5) respuestas exageradas de sobresalto

E. Estas alteraciones (síntomas de los criterios $B, C, y$ ) se prolongan más de 1 mes.

F. Estas alteraciones provocan malestar clínico significativo o deterioro social, laboral o de otras áreas importantes de la actividad del individuo.

Especificar si:

Agudo: si los síntomas duran menos de tres meses.

Crónico: si los síntomas duran tres meses o más

Especificar si:

De inicio demorado: entre el acontecimiento traumático y el inicio de los síntomas han pasado como mínimo 6 meses.

\section{Propósito:}

El presente trabajo tiene como objetivo evaluar algunas propiedades psicométricas de la escala de Trauma de Davidson. Para ello, se propone determinar la confiabilidad, la estructura factorial y la validez convergente y divergente de la escala.

\section{Método}

\section{Participantes}

Se tomó una muestra de 277 individuos (194 mujeres y 81 varones). Esta muestra total se encuentra conformada a su vez por dos submuestras: una de ellas pertenece a estudiantes 
de primer y tercer año de la Facultad de Psicología de la Universidad Nacional de Córdoba; constituida por 172 sujetos (146 mujeres y 24 varones).

La otra sub-muestra fue conformada por 105 sujetos (48 mujeres y 57 varones) pertenecientes a los siguientes establecimientos educativos, dos de nivel secundario (Instituto "Dr. Alexis Carrel" - 31 mujeres y 16 varones- y el Instituto E.N.E.T N ${ }^{0} 1-6$ mujeres y 30 varones-) y uno de nivel terciario (Instituto de Educación Superior "Blas Pascal" -11 mujeres y 11 varones), de la ciudad de Río Tercero, situada al sur de la Capital de la provincia de Córdoba.

Los sujetos que conformaron ambas muestras fueron seleccionados de manera accidental. Para la administración de los instrumentos de autoinforme, se informó a los mismos acerca del objetivo de la investigación (consentimiento informado). Su participación fue voluntaria y anónima.

\section{Instrumentos:}

En el presente estudio se utilizaron los siguientes instrumentos psicométricos de autoadministración:

Escala de Trauma de Davidson (DTS)(Davidson JRT, Book SW, Colket JT, Tupler LA, Roth S, David D y cols; 1997): esta escala está constituida por 17 ítems que corresponden y evalúan a cada uno de los 17 síntomas pertenecientes a los criterios $\mathrm{B}, \mathrm{C}$ y D, recogidos del DSM IV para el diagnóstico de Trastorno por Estrés Postraumático. Se trata de una escala de autoinforme, en la que el sujeto debe puntuar dos aspectos de cada ítem: la frecuencia de presentación y la gravedad utilizando una escala Likert de 5 puntos (de 0, nunca o gravedad nula, a 4, a diario o gravedad extrema). El marco de referencia temporal que los sujetos deben considerar es la semana previa.

Cuestionario de Acontecimiento Traumáticos (CAT) (Davidson JRT, Hughes D, Blazer DG; 1990): esta escala está conformada por un listado de 17 experiencias traumáticas más frecuentes a las que el sujeto ha de ir respondiendo si alguna vez las sufrió en su vida. Si no ha sufrido ninguno de los acontecimientos del listado, pero sí otro, tiene un espacio reservado para anotar la naturaleza del mismo.

Escala revisada del Impacto del Acontecimiento (IES - R) (Weiss DS, \& Marmar CR.; 1997): consta de 22 ítems, 15 de los cuales evalúa dos de los tres criterios 
sintomatológicos del trastorno por estrés postraumático (intrusión y evitación). Weiss y Marmar (1997) incorporaron 7 ítems más para evaluar los síntomas de hiperactivación.

Se trata de una escala de autoinforme en la que el sujeto califica el malestar o estrés generado por cada uno de los ítems. Es una escala tipo Lickert en la que los valores oscilan entre 0 (nada en absoluto) y 4 (extremadamente). El marco de referencia temporal que los sujetos deben considerar es la semana previa.

Inventario para Depresión de Beck (BDI) (Beck AT y cols.; 1978): este inventario consta de 21 ítems que indagan acerca de los síntomas cognitivos, físiológicos y conductuales de la depresión. Se trata de una escala de autoinforme en la que el sujeto valora cada ítem de acuerdo al malestar que le produce. Consiste en una escala tipo Lickert en la que los valores oscilan entre 0 y 3.

State-Trait Anxiety Inventory (STAI) (Spielberger, Gorsuch, Lushene, Vagg, Jaccobs, 1993): consiste en dos escalas de 20 ítems cada una que apuntan a medir el estado de ansiedad caracterológico y situacional de quien responde, a partir de un puntaje que puede variar entre 0 y 4 . La escala de estado pregunta a los respondientes "como se sienten ahora mismo" y la escala de rasgo pregunta como se sienten "generalmente".

\section{Resultados}

\section{Confiabilidad}

Cuando se alude a la confiabilidad de una medición, en la medida en que esta pueda repetirse y que cualquier influencia aleatoria tienda a producir medidas diferentes; constituya una fuente de error de medición.

A cada prueba se la considera como una muestra de reactivos tomados al azar de un dominio, y el error de medición se presenta sólo en la medida en que el tamaño de la muestra sea limitado. Por lo tanto si la correlación promedio entre los reactivos (ítems) es positiva, la prueba más larga será la más confiable.

En esta argumentación se entiende que la principal fuente de error de medición se debe al muestreo de reactivos. El coeficiente alfa de Cronbach sería la medida correcta de confiabilidad. El mismo arrojó un $\alpha=.89$ para el total de la escala. El coeficiente de correlación inter-ítem observado fue de $\mathrm{r}=.33$. 
Los valores de confiabilidad por consistencia interna arrojados en este estudio fueron ampliamente aceptables considerando la sugerencia de Nunnally (1991) que refiere a resultados promisorios cuando la confiabilidad de las pruebas en Ciencias Sociales y Humanas supera los valores de .80 .

Por otra parte, los valores obtenidos en esta población son similares a los obtenidos por los autores de la Escala de Trauma de Davidson (DTS) en otros estudios (Davidson y cols., 1997; Bobes y cols., 2000)

\section{Análisis Factorial}

El análisis de componentes principales realizado permitió extraer cuatro factores. Posteriormente se realizó la rotación varimax para obtener un claro patrón de cargas. (ver tabla 1)

Tabla 1: Factores rotados para la escala de Trauma de Davison Eigenvalues

Extraction: Principal Components

\begin{tabular}{ccccc}
\hline Value & Eigenvalue & \% total Variance & $\begin{array}{c}\text { Cumul. } \\
\text { Eigenvalue }\end{array}$ & Cumul \% \\
\hline 1 & 6.537576 & 38.45633 & 6.53758 & 38.45633 \\
2 & 1.651556 & 9.71504 & 8.18913 & 48.17137 \\
3 & 1.390307 & 8.17827 & 9.57944 & 56.34964 \\
4 & 1.155757 & 6.79857 & 10.73520 & 63.14821 \\
\hline
\end{tabular}

El primer factor explica el $38.45 \%$ de la varianza total y fue denominado "Reexperimentación e hiperactivación". El segundo factor explica el $9.71 \%$ del total de la varianza y fue denominado "Evitación". El tercer factor explica el $8.17 \%$ del total de la varianza y fue denominado "Embotamiento emocional" y el cuarto factor explica el 6.79 $\%$ del total de la varianza y fue denominado "Arousal".

Los resultados obtenidos de la extracción de componentes principales para la Escala de Trauma de Davidson (DTS) son alentadores. La estructura factorial que explica el constructo muestra resultados semejantes a los obtenidos previamente por otros autores en investigaciones psicométricas de la Escala de Trauma de Davidson (Cía, 2001).

Por otra parte, es sabido que la extracción de componentes principales en un análisis factorial es realizada computacionalmente de un modo secuencial, siendo el primer factor el que posee mayor porcentaje de la varianza y los siguientes con menor varianza. Lo importante de destacar en este hecho es la particularidad de que esta extracción fue realizada y es concordante con la secuencia sintomática descripta en el DSM-IV para el 
Trastorno por Estrés Postraumático (TEPT) (véase criterios diagnósticos p.p: 4 y 5). De este modo, se puede constituir como evidencia relativa de las bondades del diseño y construcción de la escala, aún careciendo del conocimiento detallado de las particularidades de la etapa original de la selección de ítems y de su implementación.

El resultado de la rotación Varimax determinó como primer factor a aquel que contenía un agrupamiento de ítems que refieren al elemento principal del Trastorno por Estrés Postraumático (TEPT) que es la re-experimentación y consecuente hiperactivación relacionada al trauma (Davidson, 1997). Conceptualmente esto cobra importancia a la hora de sustentar empíricamente las propiedades psicométricas de la Escala de Trauma de Davidson (DTS). Los demás factores que explican el constructo, si bien son elementos comunes con otros trastornos por ansiedad, son necesarios para configurar el síndrome mental descrito en el DSM-IV. Por otra parte estos hallazgos replican los de investigaciones previas respecto a las propiedades psicométricos de la Escala de Trauma de Davidson (Davidson et al.., 1997).

\section{Validez Convergente}

La validez convergente de la escala de trauma de Davidson (DTS), fue verificada mediante la observación de sus correlaciones con la escala "Impact of Event Scale Revised” (IES-R) y el "Cuestionario de Acontecimientos Traumáticos” (CAT).

La correlación registrada entre el Cuestionario de Acontecimientos Traumáticos (CAT) y la escala de trauma de Davidson (DTS) fue de $r=.23(\mathrm{p}<0.05)$, es decir, una correlación baja (ver tabla 2). Este hecho podría ser atribuido a las características de respuesta del primero, siendo estas de tipo dicotómico. Atribuir a otros factores los resultados obtenidos sería aventurado ya que carecemos en nuestro medio de investigaciones sobre las propiedades psicométricas del instrumento utilizado.

La correlación observada entre la escala revisada del impacto del acontecimiento (IES-R) y la escala de trauma de Davidson (DTS) fue de $r=.56(\mathrm{p}<0.05)$, es decir, una correlación moderada (ver tabla 2). Este resultado sustenta la validez convergente con la DTS. Davidson (1997) obtuvo con la misma escala una correlación de $\mathrm{r}=.64$. 


\section{Validez Divergente}

Para determinar la validez divergente, fueron utilizadas las escalas "State Trait Anxiety Inventory I" y "State Trait Anxiety Inventory II" (STAI-I y STAI- II) y el "Beck Depression Inventory" (BDI). Cabe destacar que la divergencia de estas escalas debe ser relativizada dado que la ansiedad evaluada por las STAIs y la depresión evaluada por el BDI, han sido reportados como emociones o trastornos comórbidos con el Trastorno por Estrés Postraumático (Davidson y Foa, 1991).

Las correlaciones obtenidas con las STAI- I y STAI -II, fueron:

- $\quad$ STAI - I con DTS: $r=.02(\mathrm{NS})$

- $\quad$ STAI - II con DTS: $r=.08(\mathrm{NS})$

La correlación observada con el BDI fue: $r=.47(p<0.05)$, es decir una correlación moderada entre las escalas (ver tabla 2). Además este resultado sugiere una correlación moderada entre el trastorno por estrés postraumático y la depresión, lo cual tiene sustento en investigaciones que han resaltado la vinculación que existe entre los eventos estresantes de carácter crónico (esto es cuando el evento traumático no ha sido reciente) y los estados depresivos, por un lado, y entre los eventos estresantes de tipo agudo y los estados ansiogénicos, por otro (Seligman, 1975; Beck y cols., 1978; Wells, 1998).

En el caso de las medidas de ansiedad (ver tabla 2), no se evidenció relación alguna, sugiriendo por un lado, la validez divergente y por otro, la relación entre los eventos agudos y crónicos descriptos precedentemente.

Tabla 2 Correlaciones registradas entre los instrumentos. Muestra de estudiantes

\begin{tabular}{|c|c|c|c|c|c|c|c|c|c|c|c|}
\hline & BDI & STAI I & STAI II & CAT & DTS F & DTSG & DTS 1 & DTS 2 & DTS 3 & DTS 4 & IES-R \\
\hline BDI & - & .04 & .15 & .07 & $.47 *$ & $.46^{*}$ & $.47 *$ & $.26^{*}$ & $.51^{*}$ & $.43^{*}$ & $.36^{*}$ \\
\hline STAI I & & - & $.77 *$ & $.23^{*}$ & .02 & .05 & .09 & -.05 & .02 & .16 & -.02 \\
\hline STAI II & & & - & .14 & .08 & .11 & .12 & -.01 & .09 & $.25^{*}$ & .05 \\
\hline CAT & & & & - & .18 & $.23 *$ & $.19^{*}$ & $.21 *$ & .17 & .13 & -.05 \\
\hline DTS F & & & & & - & $.95 *$. & $.91 *$ & $.82 *$ & $.85^{*}$ & $.83^{*}$ & $.56^{*}$ \\
\hline DTS G & & & & & & - & $.90^{*}$ & $.81 *$ & $.86^{*}$ & $.81 *$ & $.51 *$ \\
\hline DTS 1 & & & & & & & - & $.64^{*}$ & $.80^{*}$ & $.75^{*}$ & $.55^{*}$ \\
\hline DTS 2 & & & & & & & & - & $.51 *$ & $.67 *$ & $.49^{*}$ \\
\hline DTS 3 & & & & & & & & & - & $.62 *$ & $.35^{*}$ \\
\hline DTS 4 & & & & & & & & & & - & $.53 *$ \\
\hline IES-R & & & & & & & & & & & - \\
\hline
\end{tabular}




\section{Discusión}

En cuanto a las propiedades psicométricas de la Escala de Trauma de Davidson (DTS), se puede concluir que:

La confiabilidad, en su dimensión de la consistencia interna, fue verificada mediante el procedimiento alfa de Cronbach y se obtuvieron resultados muy adecuados y semejantes a los informados por los autores de la escala.

El análisis factorial permitió obtener una estructura interna de la prueba que se corresponde con la descripción sintomatológica que realiza el DSM-IV para el trastorno por estrés postraumático. Este resultado constituye un indicador de las bondades del diseño de la escala.

Entre la escala de trauma de Davidson (DTS) y la escala revisada del impacto del acontecimiento (IES-R), se observó una correlación moderada. Las conclusiones al respecto son relativas ya que no se cuenta con instrumentos validados en nuestro medio y esto impide llegar a conclusiones de mayor sustento empírico.

Con relación a la validez divergente puede concluirse que la misma quedó demostrada. Sin embargo, la divergencia entre las escalas debe relativizarse debido a que la ansiedad (evaluada por los STAIs) y la depresión (evaluada por el BDI) son trastornos comórbidos.

Como conclusión final la Escala de Trauma de Davidson (DTS) cuenta con propiedades psicométricas alentadoras para evaluar el Trastorno por Estrés Postraumático, claro está que no sin antes realizar las investigaciones necesarias para su utilización responsable.

Un aspecto de la validez de constructo que parecería ser crucial para una escala como la estudiada, es la validez discriminante. La misma podría estudiarse mediante la contrastación de dos muestras de sujetos, una constituida por sujetos de control (sin el trastorno) y otra por sujetos con la sintomatología propia del trastorno por estrés postraumático, atendiendo, además, a los criterios diagnósticos mencionados para el mismo en el DSM IV.

El criterio de selección de las muestras podría realizarse mediante la administración de otro instrumento psicométrico que evalúe los síntomas del trastorno, o bien a través del juicio de clínicos expertos. 


\section{Referencias}

American Psychiatric Association (APA) (1994). Diagnostic and statistical manual of mental disorders ( $4^{\mathrm{a}}$ ed.) (DSM-IV). Washington, DC: APA.

Beck, A.T, Rush A.J., Shaw B. F., Emery, G. (1983). Beck depression Inventory. En Terapia cognitiva de la depresión. Bilbao: Desclee de Brower.

Bobes, J. y cols. (2000). Evaluation of the psycometric properties of the Spanish version of 5 questionnaires for the evaluation of post-traumatic stress syndrome. Actas Esp. Psiquiatr 28(4): 207-218.

Breslau N., Davis G.C., Andreski P., Oeterson E. (1991). Traumatic events and posttraumatic stress disorder in an urban population of young adults. Arch Gen Psychiatry; 48: 216-222.

Cía A. H. (2001). Instrumentos Diagnósticos. En: Trastorno por Estrés Postraumático. $1^{\mathrm{a}}$ Ed. Buenos Aires: Imaginador.

Davidson J.R.T, Book SW, Colker JT y cols. (1997). Assessment of a new self- rating Scale for posttraumatic stress disorder. Psychol Med; 27: 153-160.

Davidson J.R.T, Hughes D., Blazer D.G. (1990). Traumatic experiences in Psychiatric outpatients. J Trauma Stress; 3: 459-475.

Davidson J.R.T. y Foa E.B.(1991). Diagnostic issues in posttraumatic disorder: Considerations for the DSM-IV. Journal of Abnormal Psychology; 100(3): 346-355.

Escalona R., Tupler L.A., Saur C.D., Krishnan K.R., Davidson J.R. (1997). Sceeninig for trauma history on an inpatient affective disorders unit: a pilot study. J Trauma Stress; 10(2): 299-305.

Giel R. (1990). Psychosocial processes in desasters. International Journal of Menthal Health; 19: 7-20.

Helzer J.E., Robins L.E .\& McEvoy L. (1987). Posttraumatic stress disorder in the general population. Findings of the Epidemiological Catchment Area Survey. N Engl J Med; 317: 1630-1634.

Kessler R.C., Sonnega A., Bromet E.; Hughes M.\& Nelson C.B. (1995). Posttraumatic stress disorder in the National Comorbidity Survey. Arch Gen Psychiatry; 52: 10481060 .

Evaluar.

2003, $\mathbf{n}^{\circ} 3$ (julio) 
Nunnally, J., (1991). Teoría psicométrica. Trad. de E. M. González. México: Trillas.

Seligman MEP. (1975). Helplessness: on depression, development and death.

San Francisco, EEUU: Freeman..

Spielberger CD., Gorsuch RL., Lushene R., Vagg PR y Jaccobs GA., 1993. Manual of State Trait Anxiety Inventory (Form I); CA, EEUU.: Consulting Psychologist Press.

Weiss D, \& Marmar C. (1997). Impact of Event Scale - Revised. En: Wilson J \& Keana TM (Eds.), Assessment of PTSD. New York, EEUU: Guilford.

Wells, A. (1998) Cognitive therapy of anxiety disorders. University of Manchester. John Wiley y Sons.

Wotman C.B. \& Silver R.C. (1989). The myths of coping with loss. Journal of Consulting and Clinical Psychology; 57: 349-357. 


\section{Anexo}

\section{Escala de Trauma de Davidson DTS}

Cada una de las siguientes preguntas se refiere a sucesos específicos, que usted puede haber experimentado durante la última semana. Para cada pregunta tenga en cuenta cuántas veces le ha sucedido (frecuencia) y con cuánta intensidad (gravedad). Escriba en los recuadros de al lado de cada pregunta un número de 0 a 4 , para indicar la frecuencia y la gravedad.

\begin{tabular}{ll}
\hline Frecuencia & Gravedad \\
\hline $0=$ nunca & $0=$ nada \\
$1=$ a veces & $1=$ leve \\
$2=2-3$ veces & $2=$ moderada \\
$3=4-6$ veces & $3=$ marcada \\
$4=$ a diario & $4=$ extrema
\end{tabular}

1. He tenido alguna vez imágenes, recuerdos o pensamientos dolorosos del acontecimiento.

2. He tenido alguna vez pesadillas sobre el acontecimiento.

3. He sentido que el acontecimiento estaba ocurriendo de nuevo, como si lo estuviera reviviendo

4. Hay cosas que me lo han hecho recordar.

5. He tenido sensaciones físicas por recuerdos del acontecimiento. (Como transpiración, temblores, palpitaciones, mareos, náuseas o diarrea)

6. He estado evitando pensamientos o sentimientos sobre el acontecimiento.

7. He estado evitando hacer cosas o estar en situaciones que me recordaran el acontecimiento.

8. He sido incapaz de recordar partes importantes del acontecimiento.

9. He tenido dificultad para disfrutar de las cosas.

10. Me he sentido distante o alejado de la gente.

11. He sido incapaz de tener sentimientos de tristeza o de afecto.

12. He tenido dificultad para imaginar una vida larga y cumplir mis objetivos.

13. He tenido dificultad para iniciar o mantener el sueño.

14. He estado irritable o he tenido accesos de ira.

15. He tenido dificultades para concentrarme

16. Me he sentido nervioso, fácilmente distraído, o como "en guardia".

17. 17. He estado nervioso o me he asustado fácilmente 